\title{
Temperature Compensated Internal LNA Noise Calibration Source
}

\author{
Jun Shi, Member, IEEE and Sander Weinreb, Life Fellow, IEEE
}

\begin{abstract}
In this letter, a new temperature compensated internal LNA noise calibration source for system temperature monitoring is presented. It is easily integrated into amplifiers and has a negligible impact on the noise performance. The power level of the proposed calibration source is temperature compensated by a negative temperature coefficient (NTC) thermistor varying the bias voltage of the noise diode. The measured variation of the compensated calibration level is only $\pm 0.5 \mathrm{~K}$ for $34 \mathrm{~K}$ calibration level at $1.4 \mathrm{GHz}$ in a temperature range from $-40{ }^{\circ} \mathrm{C}$ to $+40{ }^{\circ} \mathrm{C}$. This internal noise calibration source is controlled by the detection of a tone signal applied on the RF output cable thus requiring no additional wiring to the amplifier. The designed noise calibration source provides monitoring of system noise temperature without the additional noise and cost of a directional coupler between ambient-temperature LNA and antenna.
\end{abstract}

Index Terms - Calibration, noise diode, LNA, temperature compensation, low noise, receiving system.

\section{INTRODUCTION}

$\mathrm{V}$ ERY low noise receiving systems as used in radio astronomy or space communications often utilize a noise calibration source to monitor the system noise temperature which may vary due to atmospheric noise, RFI, changes in the LNA, or earth-radiation dependent upon antenna pointing [1]. The most common method for this monitoring is through a directional coupler and noise source between the LNA and antenna as shown in Fig. 1(a) [2]. When the noise source is on, the added system noise due to the coupling is defined as the calibration level $\left(T_{\text {cal }}\right)$. The directional coupler introduces a loss of approximately $0.2 \mathrm{~dB}$ in the low microwave range [3], [4]. This is acceptable in most cryogenic radio astronomy receivers [5], [6], since the coupler at $20 \mathrm{~K}$ physical temperature only adds $1 \mathrm{~K}$ to the system noise.

Recently, a $7 \mathrm{~K}$ noise room temperature LNA has been developed [7] and provides a high-performance receiving system without the costly cryogenic systems. For the noncryogenic environment, the directional coupler is no longer acceptable since the $0.2 \mathrm{~dB}$ loss will introduce $14 \mathrm{~K}$ to the system noise at room temperature. Another method to monitor the system noise is by the Y factor measurement with absorber material covering the feed aperture as the hot load and sky as the cold load [8], [9]. The disadvantage of the method is that it requires human or motorized interaction to place the absorber and is not easily implemented.

Manuscript received March 12, 2021; received April 30, 2021; accepted May 23, 2021. Date of publication xxxx, 2021.

J. Shi (shijun@caltech.edu) and S. Weinreb (sweinreb@caltech.edu) are with California Institute of Technology, Pasadena, CA, 91125

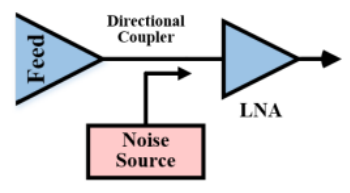

(a)

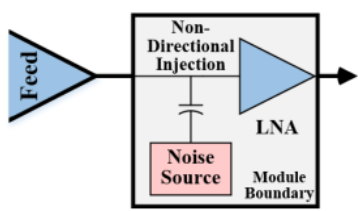

(b)
Fig. 1. Block diagram of part of a radio astronomy receiver (a) with a directional coupler and (b) with presented non-directional injection.

This letter considers a simpler calibration method utilizing a noise current source injected into the LNA as shown in Fig. 1(b) with considerations as follows.

1) When the calibration is off, the noise added to the LNA should be small, less than $1 \mathrm{~K}$. When the calibration is on, the added noise should be comparable to the expected system temperature, $T_{\text {sys }}$, of the order of $35 \mathrm{~K}$ to give low statistical fluctuation to the $T_{\text {sys }}$ measurement with the Y factor method [9], [10].

2) Integration of the noise sources into the LNA raises questions of how it will be powered and controlled. A method of accomplishing this by the DC voltage and a tone on the RF output coaxial cable is described in Section II.

3) The temperature variation of the noise calibration versus temperature is important as the LNA is usually located in an outdoor environment with large temperature variation. Temperature compensation of the noise source could be performed with a feedback loop [11]. We provide a simpler method described in Section II.

4) The non-directional injection shown in Fig. 1(b) causes the injected noise to be a function of antenna impedance and LNA input impedance. This dependence is small if the antenna is matched to $50 \Omega$ and the effect is quantified in Section III.

The noise source has been installed on 18 LNAs [7] with variation among units and temperature described in Section IV.

\section{Circuit DESIGN DESCRIPTION}

The avalanche noise diode is commonly used for the noise source design [12-16]. We selected a Noisecom NC302L because of its relatively low avalanche voltage (less than $+8 \mathrm{~V}$ ) and beam lead packaging convenient for integration into an LNA [17]. The diode noise excess noise ratio (ENR) is in the 30 to $35 \mathrm{~dB}$ range which is a noise temperature greater than $10^{5}$ 


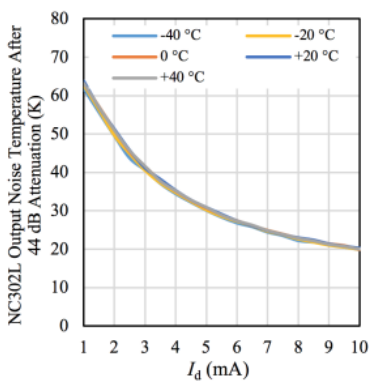

(a)

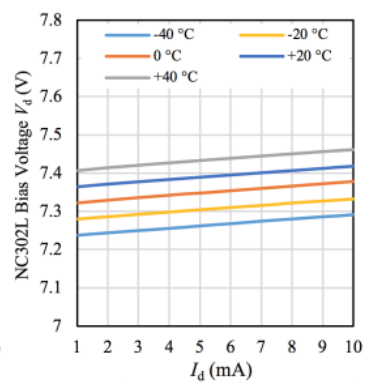

(b)
Fig. 2. (a) Measured output of NC302L as a function of diode current and temperature. Level shown is after $44 \mathrm{~dB}$ of attenuation. (b) Measured NC302L bias voltage as a function of diode current and temperature.

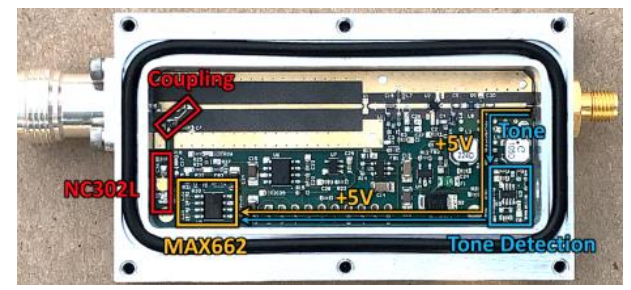

(a)

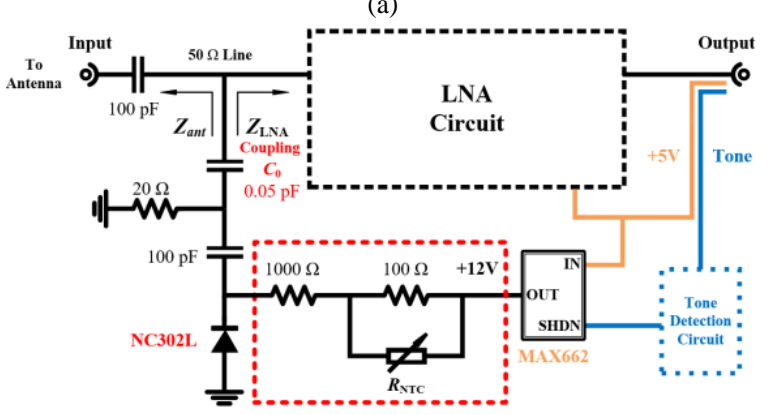

(b)

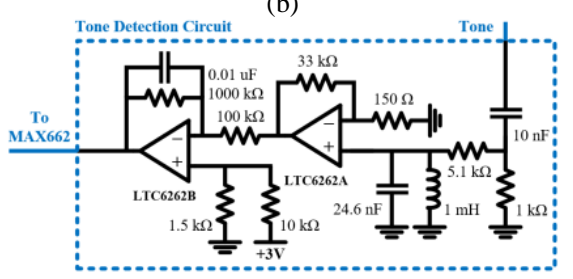

(c)

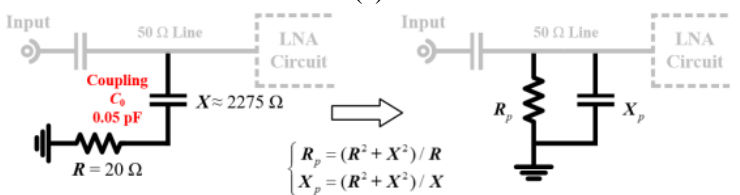

(d)

Fig. 3. (a) Internal view of the custom LNA. (b) Schematic showing the input circuit with coupling to a noise diode that is powered from the $+12 \mathrm{~V}$ boost converter. (c) Schematic showing the tone detection circuit. (d) The equivalent shunt resistance of the series $0.05 \mathrm{pF}$ and $20 \Omega$.

$\mathrm{K}$. Thus, the diode can be regarded as a large noise source and can be weakly coupled to the LNA input to satisfy criteria 1) above. The measured noise temperature (after $44 \mathrm{~dB}$ of attenuation) versus diode temperature and current $\left(I_{\mathrm{d}}\right)$ is shown in Fig. 2(a), and the corresponding bias voltage $\left(V_{\mathrm{d}}\right)$ versus temperature and current $\left(I_{\mathrm{d}}\right)$ is shown in Fig. 2(b). According Fig 2(a), when we drive the NC302L with a constant current, the output noise will change little with the temperature. This constant vs temperature can be realized by compensating the diode voltages vs temperature as described next.

The noise calibration source was integrated into the custom LNA [7] as shown in Fig. 3(a). A complete schematic of the

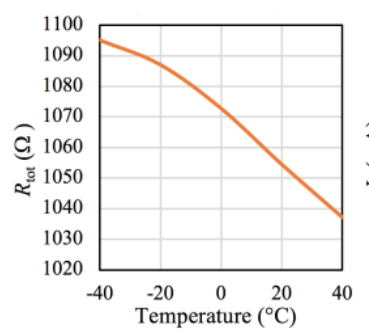

(a)

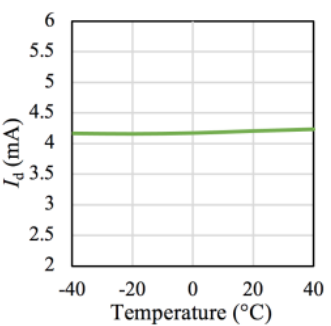

(b)
Fig. 4. (a) The total resistance $R_{\text {tot }}$ versus temperature. (b) The derived diode current $I_{\mathrm{d}}$ versus temperature.

noise source is shown in Fig. 3(b). It was desirable to power the noise diode from the $+5 \mathrm{~V}$ utilized to power the LNA, and a MAX662, $+5 \mathrm{~V}$ to $+12 \mathrm{~V}$ boost converter [18], was selected on the basis that it utilizes capacitor charge-pumps. Many boost converters use inductors to achieve the boost and were avoided because it would be difficult to isolate the LNA circuitry from the inductive coupling. The requirement for constant current versus temperature could be met with a current regulator between the noise diode and the $+12 \mathrm{~V}$ source. However, it is much simpler to utilize a negative-temperature-coefficient (NTC) resistor [19] to produce the required bias voltage versus temperature shown in Fig. 2(b) to stabilize the diode current and hence the noise output. The three resistors to accomplish this are shown in the red dashed box in Fig. 3(b), and their total resistance $\left(R_{\mathrm{tot}}\right)$ and the derived diode current $\left(I_{\mathrm{d}}\right)$ are shown in Fig. 4 . Here, $R_{\mathrm{tot}}=1000+R_{\mathrm{NTC}} \times 10 /\left(R_{\mathrm{NTC}}+10\right) \Omega$ where $R_{\mathrm{NTC}}$ is given by [19], and $I_{\mathrm{d}}$ is derived from the formula $V_{\mathrm{d}}+R_{\mathrm{tot}} \times I_{\mathrm{d}}=$ $12 \mathrm{~V}$ where the relation between $V_{\mathrm{d}}$ and $I_{\mathrm{d}}$ is given by Fig. 2(b).

As shown in Fig. 3(b), the weak coupling of the noise diode is accomplished through $C_{0}$. The value of $C_{0}$ is $0.05 \mathrm{pF}$ and is available as an off-the-shelf surface-mount chip capacitor. At the desired LNA operating frequency of $1.4 \mathrm{GHz}, C_{0}$ presents a reactance $X \approx 2275 \Omega$ and is in series with a $20 \Omega$ load. When the NC302L is off, the noise contributed by the $20 \Omega$ load can be calculated by finding the equivalent shunt resistance $R_{\mathrm{p}}$ of the series RC shown in Fig. 3(d) and comparing this with the 50 $\Omega$ generator impedance. The shunt resistance is about 260000 $\Omega$ which adds noise of $50 / 260000 \times 300 \mathrm{~K}=0.06 \mathrm{~K}$, thus easily meeting the requirement of $<1 \mathrm{~K}$ added noise with the noise diode off. Due to the high reactance and the very low added noise, the coupling circuit has no impact on the LNA matching and can be considered independent of the LNA. The $20 \Omega$ load as well as the diode current are selected to give the desired calibration level which is approximately $35 \mathrm{~K}$.

Switching the noise calibration source from on to off is accomplished through the SHDN (shut down) pin of the boost converter (MAX662); when SHDN is low the $+12 \mathrm{~V}$ is on and when SHDN is high the $+12 \mathrm{~V}$ is off. The switching transients generated by the converter charge pump are off thus assuring no interference with the LNA when the calibration signal is off. The SHDN pin is controlled by a $32 \mathrm{kHz}$ tone applied on the LNA RF output connector thus requiring no additional wiring to the amplifier which is good for RFI proofing for LNAs used in arrays. This tone, when present with amplitude $>200 \mathrm{mV}$ peak-to-peak, forces the SHDN pin to low. As shown in Fig. $3(\mathrm{c})$, the filtering and detection of the tone is accomplished with a LTC6262 dual operational amplifier which sets a threshold so that SHDN is clearly on or off and the noise calibration is not dependent upon the tone amplitude. 


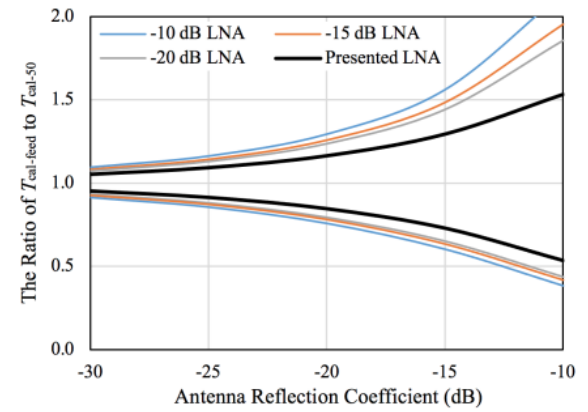

Fig. 5. The simulated range of the ratio $T_{\text {cal-feed }} / T_{\text {cal-50 }}$ dependent upon the antenna and LNA reflection coefficient magnitudes is shown above.

\section{Modeled Possible RANGe of CALIBRATION with ANTENNA MISMATCH}

It is assumed that the calibration level $\left(T_{\text {cal }}\right)$ of the internal calibration source will be first measured with a well-matched $50 \Omega$ noise source connected to the LNA input $\left(T_{\text {cal-50) }}\right)$. When the LNA is connected to an antenna feed, the effective calibration level ( $\left.T_{\text {cal-feed }}\right)$ will change due to an impedance different than $50 \Omega$ presented by the antenna. The $T_{\text {cal-feed }}$ can be determined by connecting the LNA to the feed and performing a $\mathrm{Y}$ factor measurement with absorber material covering the feed aperture as the hot load and sky as the cold load [8], [9]. This $T_{\text {cal-feed }}$ calibration needs only be performed once, is expected to be very stable, and can be used to monitor the system noise temperature as a function of time. Note that when we install the noise source in a different LNA, the calibration level will change due to the LNA impedance change as well.

However, it is of interest to predict the range of the ratio $T_{\text {cal- }}$ feed $/ T_{\text {cal-50 }}$ as a function of only the antenna and LNA reflection coefficient magnitudes. This was performed using AWR/MWO circuit analysis software using the schematic shown in Fig. 3(b). The injected noise can be considered as a current source injected into the connection of LNA and antenna. The voltage at this node is then equal to the current divided by the sum of admittances of the LNA and antenna which can be measured with a network analyzer. This has been calculated by varying the admittance (amplitude and phase) of the antenna and LNA to find the maximum and minimum of the ratio. The simulated results at $1.4 \mathrm{GHz}$ are shown in Fig. 5 for all $-10 \mathrm{~dB},-15 \mathrm{~dB}$, and $-20 \mathrm{~dB}$ LNAs as well as the presented LNA. For example, for all $-20 \mathrm{~dB}$ antennas and $-15 \mathrm{~dB}$ LNAs, the ratio $T_{\text {cal-feed }} / T_{\text {cal- }}$ 50 is between 0.78 and 1.26 .

\section{EXPERIMENTAL RESUlTS}

The noise calibration source has been fabricated and the noise performance was measured with a noise figure analyzer (N8975A, Keysight) and a calibrated noise source [20] (the reflection coefficient is lower than $-30 \mathrm{~dB}$ ). During the measurement, the LNA was placed inside a temperature chamber (SU-241, ESPEC) capable of reaching stable temperatures of $-40{ }^{\circ} \mathrm{C}$ to $+40{ }^{\circ} \mathrm{C}$. The output of the LNA was connected to a bias tee to provide the DC power and the $32 \mathrm{kHz}$ tone for control of the internal noise calibration source. The calibration level measurement versus frequency was performed at five temperatures from $-40{ }^{\circ} \mathrm{C}$ to $+40{ }^{\circ} \mathrm{C}$ with results plotted

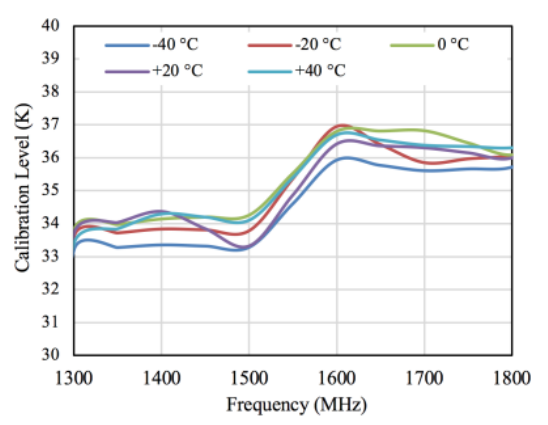

Fig. 6. Measured calibration level vs frequency at five different temperatures.

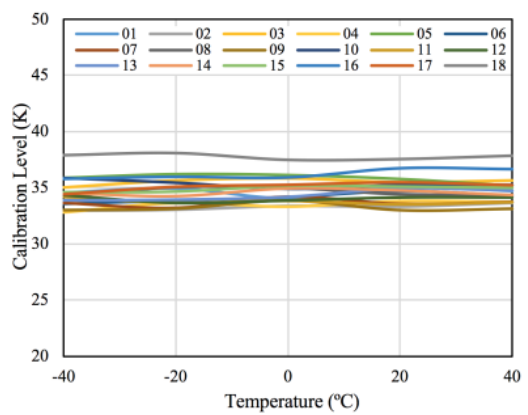

Fig. 7. Calibration levels at $1.4 \mathrm{GHz}$ for $18 \mathrm{LNAs}$ in the temperature range from $-40{ }^{\circ} \mathrm{C}$ to $+40{ }^{\circ} \mathrm{C}$. The variation from one unit to another is due to differences in the noise diodes. For any one of the 18 LNAs the calibration level vs temperature varies by less than $\pm 0.5 \mathrm{~K}$ from the mean value.

in Fig. 6. In the figure, the $T_{\text {cal }}$ curves vs frequency are similar at all the 5 temperatures from $-40{ }^{\circ} \mathrm{C}$ to $+40{ }^{\circ} \mathrm{C}$, and the variation with temperature is less than $\pm 0.5 \mathrm{~K}$ from the mean calibration level of approximately $34 \mathrm{~K}$ at $1.4 \mathrm{GHz}$. A repeat of the measurement with temperature compensation disabled gave $\pm 3 \mathrm{~K}$ variation of the calibration level. The measured data changes little within a 12-hour period.

As is typical with avalanche noise diodes, the ENR values can differ from diode to diode. To investigate this and to check the repeatability of the design, the calibration level of 18 different copies of the circuit including LNA were measured with results shown in Fig. 7. The figure shows a variation of \pm 3 $\mathrm{K}$ from the mean of $35 \mathrm{~K}$ and all 18 copies exhibit the $\pm 0.5 \mathrm{~K}$ stability with temperature.

\section{CONCLUSIONS}

The design and test results of a novel noise calibration system have been presented. The system has the following new features compared to previous works: 1) The noise calibration source is simple and compact so it can be easily integrated into an LNA. The system adds less than $0.1 \mathrm{~K}$ to the LNA noise when the calibration is off; 2) The noise calibration source requires no additional wires to the LNA. It is powered from the same $+5 \mathrm{~V}$ used for the LNA DC bias and is controlled by a tone applied to the RF output cable; 3) Elimination of a directional coupler eliminates the coupler noise as well as the cost and size of the coupler; 4) The change in calibration level due to nondirectional coupling with antenna mismatch has been quantified. This change is stable with time and does not affect the application of the internal source to monitor the stability of the system noise temperature with time; 5) The calibration level has been temperature compensated to a level of $\pm 1.5 \%$ stability over the temperature range of $-40{ }^{\circ} \mathrm{C}$ to $+40{ }^{\circ} \mathrm{C}$. 
Calibration Paper, Final file, May 2021

\section{REFERENCES}

[1] F. T. Ulaby, R. K. Moore, and A. K. Fung, Microwave Remote Sensing: Active and Passive, Radar Remote Sensing and Surface Scattering and Emission Theory, vol. 2. Reading, MA, USA: Addison-Wesley, 1981.

[2] E. W. Bryerton, "A Cryogenic Integrated Noise Calibration and Coupler Module Using a MMIC LNA," IEEE Trans. Microw. Theory Techn., vol. 59, no. 8, pp. 2117-2122, Aug. 2011.

[3] Data sheet, model: 4012-30, L3Harris Narda-MITEQ, NY, 11788, USA, www.nardamiteq.com.

[4] Data sheet, RFDC1G2G30, RF-Lambda USA LLC, TX, 75010, USA, www.rflambda.com.

[5] G. Valente, G. Montisci, T. Pisanu, A. Navarrini, P. Marongiu and G. A. Casula, "A Compact L-Band Orthomode Transducer for Radio Astronomical Receivers at Cryogenic Temperature," IEEE Trans. Microw. Theory Techn., vol. 63, no. 10, pp. 3218-3227, Oct. 2015.

[6] G. Valente, G. Montisci, S. Mariotti, "High-performance microstrip directional coupler for radio-astronomical receivers at cryogenic temperature," Electron. Lett., vol. 50, no. 6, pp. 449-451, Mar. 2014.

[7] S. Weinreb and J. Shi, "Low Noise Amplifier With 7-K Noise at 1.4 $\mathrm{GHz}$ and $25^{\circ} \mathrm{C}$," IEEE Trans. Microw. Theory Techn., vol. 69, no. 4, pp.2345-2351, Apr. 2021.

[8] W. J. Welch, DC-J. Bock, M. C. Fleming, and D. D. Thornton. "Amplitude calibration in Interferometry," Imaging at Radio through Submillimeter Wavelengths, vol. 217, 2000, pp. 309-315.

[9] K.O. Neil, "Single Dish Calibration Techniques at Radio Wavelength", in NAIC/NRAO school on Single Dish Radio astronomy-2001. ASP Conference Series, Vol. cs-278, San Francisco, CA, pp. 1-18.

[10] D. M. Pozar, Microwave Engineering, 2nd Ed., Toronto: McGraw-Hill, Inc., 1992, pp. 553-556.

[11] M. Oh, J. Lee and Y. Kim, "ENR compensation of a noise source using calibration coefficients with variation of the ambient temperature," IEEE Trans. Microw. Theory Techn., vol. 65, no. 6, pp. 2201-2210, Jun. 2011.

[12] F. Alimenti, G. Simoncini, G. Brozzetti, D. Dal Maistro, M. Tiebout, "Millimeter-Wave Avalanche Noise Sources Based on p-i-n Diodes in $130 \mathrm{~nm}$ SiGe BiCMOS Technology: Device Characterization and CAD Modeling,” IEEE Access, vol. 8, pp. 178976-178990, 2020.

[13] F. Alimenti, G. Tasselli, C. Botteron, P. Farine and C. Enz, "Avalanche microwave noise sources in commercial 90-nm CMOS technology," IEEE Trans. Microw. Theory Techn., vol. 64, no. 5, pp. 1409-1418, May. 2016.

[14] C. T. Coen, M. Frounchi, N. E. Lourenco, C. D. Y. Cheon, W. L. Williams, and J. D. Cressler, "A $60-\mathrm{GHz}$ SiGe radiometer calibration switch utilizing a coupled avalanche noise source," IEEE Microw. Wireless Compon. Lett., vol. 30, no. 4, pp. 417-420, Apr. 2020.

[15] J. C. Azevedo Goncalves et al., "Millimeter-wave noise source development on SiGe BiCMOS 55-nm technology for applications up to 260 GHz," IEEE Trans. Microw. Theory Techn., vol. 67, no. 9, pp. 3732-3742, Sep. 2019.

[16] H. Ghanem et al., "Modeling and analysis of a broadband Schottky diode noise source up to $325 \mathrm{GHz}$ based on 55-nm SiGe BiCMOS technology," IEEE Trans. Microw. Theory Techn., vol. 68, no. 6, pp. 2268-2277, Jun. 2020.

[17] Data sheet, NC302LBL, Noisecom, Parsippany, NJ, 07054, USA, www.noisecom.com.

[18] Data sheet, MAX662A, Maxim Integrated, CA, 95134, USA, www.maximintegrated.com.

[19] Data sheet, NTCG163EH101HT1, TDK Corporation, NY, 11556, USA, www.tdk.com.

[20] N4000 noise source, technical overview, 5988-0081, Keysight, Santa Rosa, CA, 95403, USA, pp. 7, www.keysight.com. 